TITLE:

\title{
Measurement and Analysis of Lymphocyte Deformation in Microchannel Contraction Flows Using a Compound Drop Model
}

\author{
AUTHOR(S): \\ Tatsumi, K.; Haizumi, K.; Sugimoto, K.; Nakabe, K.
}

\section{CITATION:}

Tatsumi, K.... [et al]. Measurement and Analysis of Lymphocyte Deformation in Microchannel Contraction Flows Using a Compound Drop Model. Flow, Turbulence and Combustion 2016, 96(1): 245-260

\section{ISSUE DATE:}

2016-01

URL:

http://hdl.handle.net/2433/203602

\section{RIGHT:}

The final publication is available at Springer via http://dx.doi.org/10.1007/s10494-015-9633-1; The full-text file will be made open to the public on 5 August 2016 in accordance with publisher's 'Terms and Conditions for Self-Archiving':; の論文は出版社版でありません。引用の際には出版社版をご確認じ利用ください。; This is not the published version Please cite only the published version. 
Measurement and Analysis of Lymphocyte Deformation in Microchannel Contraction Flows using a Compound Drop Model

\author{
K. Tatsumi, K. Haizumi, K. Sugimoto and K. Nakabe \\ Department of Mechanical Engineering and Science, Kyoto University, Japan
}

Corresponding author: Kazuya Tatsumi

Kyotodaigakukatsura, Nishikyo-ku, Kyoto, Kyoto 615-8540, Japan

tatsumi@me.kyoto-u.ac.jp

\begin{abstract}
To understand the physical properties of lymphocytes and to develop a numerical model that can predict their motion and deformation in flows, a three-dimensional numerical simulation of lymphocytes flowing through the contraction region of a microchannel was performed using a compound drop model. The present model considers shear-thinning effects on the cytoplasm and the nucleus by modeling a second droplet inside the primary cell. The time-dependent characteristics of the deformation index $(D I)$ of the lymphocyte, and the effects of the flow rate and the nucleus position on the $D I$ are discussed and compared with the measurement. The results demonstrated that the conventional drop model, in which Newtonian fluid properties are applied to the cytoplasm, cannot correctly predict the deformation of the cell in the contraction region, where the nonlinear effects become important, whereas the compound drop model was consistent with experimental measurements of lymphocyte deformation. The size and position of the nucleus were found to influence the shape of the lymphocyte as measured by a change in the deformation rate of the leading and trailing sides of the lymphocyte. Further, a simplified model of lymphocyte deformation in the steady elongational flow was employed to determine the apparent viscosity, including the shear-thinning effects, of the lymphocyte.
\end{abstract}

Key words: Lymphocytes, Deformability, Elongational flow, Microchannel Flow, Numerical model, Compound drop model, Immersed boundary method, Shear-thinning effect 


\section{Introduction}

Experimental and simulation-based analyses of blood flow have been instrumental in the understanding of this physical phenomenon in living organs and throughout the body, and in the use to enhance diagnosis and treatment of clinical diseases. In addition to this, current technologies are focused on single cell analyses of blood cells using micro-devices, such as Labon-a-Chip and micro-TAS (total analysis systems). Measurement of the biological and physical characteristics of each individual cell using these techniques can be performed with high accuracy, speed, compactness and low cost. The use of these approaches not only provides new insight toward the basic biology of these systems, they have the potential to make a large contribution to the field of medicine by the detection of early stage clinical diseases, including but not limited to cancer and malaria diagnostics as examples [1-3].

In order to put these analysis and technologies to practical uses, it is essential to understand the motion and deformation of a single blood cell in flows of blood vessels, medical equipment, and micro-devices. Further, it is important to develop a numerical model that can accurately simulate these behaviors of the blood cells in terms of helping get a better understanding of the cell characteristics, and aiding in the design and development of sensors, sorters, cell injectors, and microbiological assays of such micro-devices which require control of the cell position with high precision and information of the cell shape in order to achieve the high throughput while maintaining resolution during measurements.

Analysis of blood flow and cells have predominantly focused on the characterization of red blood cells (RBCs), as they influence fluid properties such as viscoelasticity as well as the generation of stress at the vessel walls [4-10]. White blood cells (WBCs) have received little attention, despite their key role in the immune system [11,12], and that defects of WBCs are associated with a number of severe diseases [13]. Characterization of the physical properties of WBCs is therefore important not only to understand the flow and transport phenomena within blood vessels, but also potentially in the diagnosis of WBC-associated diseases and developing the technologies for single cell analysis.

Measurement of white blood cell elastic properties, or deformability, have been performed previously, mostly using the micropipette aspiration technique whereby a single cell is aspirated using a pipette with a diameter smaller than the cell [14-19]. By measuring the aspiration speed of the cell and the pressure applied upstream of the pipette, the deformability can be obtained [20]. However, several problems limit this technique, including the presence of wall friction that affects the measurement accuracy, the difficulty of verifying the deformation characteristics of the lymphocytes in flow, and the fact that it is time consuming and can only be performed in low throughput.

For numerical simulations, several of approaches have been made to model the properties of 
WBCs. Luo et al. [21] modeled lymphocytes as a single drop, which were found to be equivalent to lymphocytes averaged over the whole cell when the deformation properties were determined in a straight channel with relatively low shear stress. Kan et al. [22] calculated the effect of the presence of nucleus using the compound drop model and compared the model with the micropipette aspiration method. Khismatullin and Truskey [23] have presented a leukocyte model using the compound drop, and analyzed the interaction and adhesion of the leukocyte to a wall in a straight channel flow. However, these models have not made a clear discussion on characteristics of the shear-thinning effects of the cytoplasm and the nucleus effects particularly in high flow rate flows accompanying large stresses, and perform a quantitative validation on the cell deformability and motions in flows.

In the present study, a three-dimensional numerical simulation using a compound drop model of lymphocyte deformation in a microchannel is performed with the aim to characterize the physical properties and characteristics of lymphocytes in fluids and to validate the models. The use of the microchannel allowed the shape of each cell to be measured directly, quickly and continuously, and by incorporating a contraction region, which produces an elongational flow in which the lymphocyte will deform over time, allowed measurement of the viscoelastic properties of the cell. Therefore, results of large samples with accurate data can be obtained which is essential to model the single cell. As for the models, those considering the effects of the viscous shear-thinning and the nucleus are presented in this study. These models were experimentally validated by measurements of the relationship between the lymphocyte deformation and flow rate in a microchannel contraction flow, and visualization of the nucleus. Thus, discussions are made on the effects of the flow rate and nucleus position on the deformation characteristics in the evaluation of each model and to understand the importance of the influence of the nucleus on flow effects. At the end of this article, a method using the simplified model considering the deformation characteristics of the lymphocyte in simple steady elongational flow is introduced to measure the apparent viscosity of the lymphocyte from the experimental data.

\section{Numerical methods}

\subsection{Lymphocyte model}

The basic structure of a lymphocyte consists of a cell membrane, cytoplasm, nuclear membrane, and nucleoplasm [19] (Fig. 1). To model the dynamics of a lymphocyte, the kinetic properties of each structural component are considered. The models presented in this study are based on the drop model with modification as described. The basic drop model simply consists of a cytoplasm surrounded by a membrane with zero thickness and surface tension applied, and incorporates properties of Newtonian fluids applied to the cytoplasm. This model is referred to as the ND model hereafter. Two other models are presented in this study with additional parameters applied 
in iterations of the basic model. The first one is an adaptation of shear-thinning drop (STD) model, where the shear-thinning effect of the cytoplasm is considered by incorporating viscosity as defined in Eq. (1) [24].

$$
\mu_{c}=\max \left[\mu_{c 0}\left(1+\frac{\dot{\gamma}}{\dot{\gamma}_{c}}\right)^{-\beta}, 6.25\right][\mathrm{Pa} \cdot \mathrm{s}]
$$

where $\mu_{\mathrm{c} 0}$ is the zero-shear viscosity, and $\gamma_{\mathrm{c}}$ and $\beta$ are parameters that describe the reduction in viscosity. Note that a lower limit $6.25 \mathrm{~Pa} \cdot \mathrm{s}$ is applied to $\mu_{\mathrm{c}}$ in order to prevent a significant decrease in the viscosity parameter. The other approach is the compound drop with shear-thinning effect (STCD) model. In this model, a second drop of much higher viscosity is located within the primary drop of the STD model, which represents the nucleus (inner drop) within the cytoplasm (outer drop). The initial diameters of the outer and inner drops are $15 \mu \mathrm{m}$ and $9 \mu \mathrm{m}$, respectively.
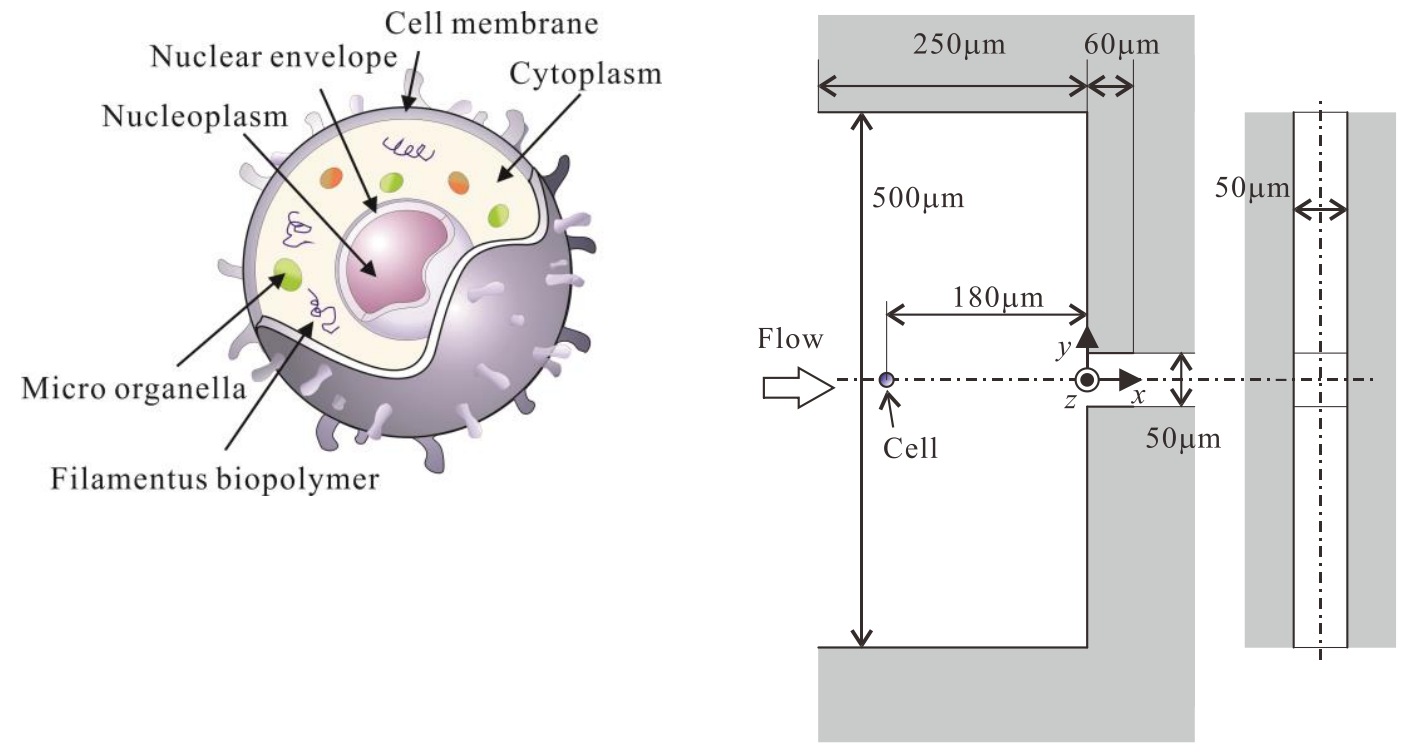

Fig. 1: Schematic of the lymphocyte structure. Fig. 2: Schematic of the contraction region in the microchannel as used in the experimental analyses and simulations, which is used as the framework on which the computation models are built. 


\subsection{Numerical procedure}

The governing equations solved in this study are the continuity and Navier-Stokes (NS) equations expressed in unsteady and incompressible forms for three-dimensional Cartesian coordinates. A finite volume method is employed to solve these equations. Second order central scheme and first order upwind schemes are used to discretize the diffusion and convection terms of the NS equation. A SIMPLER algorithm is applied to couple the continuity and NS equations to solve the pressure field.

In order to couple and calculate the conjugate problem of the lymphocyte and the flow field, an immersed boundary method [25] is applied to the boundary of the surface of the drop and the surrounding fluid. In this case, the force acting on each node of the membrane is included as an external force term in the NS equation, which is termed feedback forcing. The velocity of the fluid located adjacent to each node is also applied to the membrane nodes, and is assumed that the membrane follows the flow field. These velocities determine the motion and deformation of the lymphocyte.

As shown in Section 2.3, the viscosity of the cytoplasmic fluid in the lymphocyte differs from that of the surrounding fluids. The drastic change in viscosity at the interface of these two fluids can result in a failure of the conservation in momentum and an increase in the instability of the computation. The viscosity at the interface is changed continuously by applying the trigonometric function as shown in Eq. (2).

$$
\mu= \begin{cases}\mu_{1}+\frac{1}{2}\left\{1+\sin \left(\frac{\pi \xi}{2 \Delta x}\right)\right\}\left(\mu_{2}-\mu_{1}\right), & \left(|\xi|<\xi_{0}\right) \\ \mu, & \left(|\xi|>\xi_{0}\right)\end{cases}
$$

where $\xi$ is the distance between the grid node of the fluid and the membrane surface, and $\xi_{0}$ is the constant that defines the width of the interpolation area. $\xi_{0}$ was set as $\Delta x$, which is the grid width. $\mu_{1}$ and $\mu_{2}$ are the viscosities of the two fluids separated by the interface.

\subsection{Numerical conditions}

The computational domain is shown in Fig. 2. The contraction region is the same with the one applied to the microchannel, and is used as the framework on which the computation models were built. The inlet of the domain is located $250 \mu \mathrm{m}$ upstream from the contraction point. The coordinate origin is located at the entrance of the contraction region along the channel centerline. A Neumann boundary condition is applied at the outlet. The initial position of the lymphocyte, whose initial shape was set as a sphere, was $(x, y, z)=(-180,0,0) \mu \mathrm{m}$ relative to the coordinate origin.

The fluid and membrane properties of the cell applied to each model are summarized in Table 1. The tension applied to the membrane is set as $30 \mu \mathrm{N} / \mathrm{m}$ as described by Yeung et al. [26] and 
Needham et al. [27]. The viscosity of the cytoplasm is considered to decrease when it passes the contraction region due to high shear rate caused by the deformation of the cell. In order to match the deformation degree with the experiment in the ND model, the viscosity of the cytoplasm was defined as $10 \mathrm{~Pa} \cdot \mathrm{s}$.

Table 1: Lymphocyte properties of each drop model.

\begin{tabular}{lccc}
\hline Parameters & ND model & STD model & STCD model \\
\hline Cell membrane tension $\tau_{\mathrm{c}}[\mu \mathrm{N} / \mathrm{m}]$ & 30 & 30 & 30 \\
Apparent viscosity $[\mathrm{Pa} \cdot \mathrm{s}]$ & 10 & - & - \\
Zero shear viscosity $\mu_{\mathrm{c} 0}[\mathrm{~Pa} \cdot \mathrm{s}]$ & - & 250 & 250 \\
Shear-thinning parameter $\beta[-]$ & - & 0.5 & 0.5 \\
Characteristic shear rate $\dot{\gamma}_{c}[1 / \mathrm{s}]$ & - & - & 0.05 \\
Nuclear membrane tension $\tau_{\mathrm{n}}[\mathrm{Pa} \cdot \mathrm{s}]$ & - & - & 300 \\
Nucleoplasm viscosity $\mu_{\mathrm{n}}[\mu \mathrm{N} / \mathrm{m}]$ & - & - & 250 \\
\hline
\end{tabular}

The viscosity of the cytoplasm in the STCD model should be less than in the STD model due to the influence of the nucleus. However, as the viscosity of the cytoplasm itself is not well known, the same value for cytoplasmic viscosity in the STD model is used in the STCD model. The viscosity of the nucleus is based on a value of $2.22 \times 10^{3} \mathrm{~Pa} \cdot \mathrm{s}$ as previously described [28], however, a value $1 / 10^{\text {th }}$ of that described is used in order to obtain a stable solution in the computation. The tension of the nuclear membrane is defined based on the assumption that the ratio between the viscosity of the cytoplasm and tension on the cell membrane is equivalent to the ratio between the nucleus and the nuclear membrane [15]. Still, it is confirmed that these influences on the deformation degree of the lymphocytes are negligible in the computation although the viscosity of the nucleus and the related nuclear membrane tension are different from those of other references.

The simulated fluid is modeled on the experimental microchannel flow solution, which consisted of phosphate buffers saline (PBS) supplemented with polyvinylpyrrolidone (PVP) at $12.5 \mathrm{wt} \%$ in order to increase the fluid viscosity. Supplement of the PVP enables us to obtain a higher deformation degree at the contraction region. These values are matched with the ones measured in the experiment, which will be described shortly.

Mass flow rate $Q$ is varied in the range of $0.4-16 \mu \mathrm{L} / \mathrm{min}$. The Reynolds number is defined as $R e$ $=\rho U_{\mathrm{m}} H / \mu_{\mathrm{m}}$, where $\rho$ is the fluid density (set at $1000 \mathrm{~kg} / \mathrm{m}^{3}$ ), $U_{\mathrm{m}}$ is the cross-sectional average 
velocity of the channel inlet, $H$ is the channel height, and $\mu_{\mathrm{m}}$ is the fluid viscosity. $R e$ varies in the range of $5 \times 10^{-4}-2 \times 10^{-3}$.

A grid dependency test was carried out using the STCD model. A 20\% finer grid compared with the one employed in the computation discussed in Section 4 was used, and the cell shape at the entrance of the contraction region $(x=0)$ was examined. Under these two grid conditions, the width of the cell and nucleus in the spanwise and height directions differed by less than $1 \%$, whereas the streamwise length increased by $5 \%$ in the finer grid case. However, since this was the maximum deviation determined during the computation, the grid used in the computation is considered to be sufficient to carry out the discussion of this study.

Since the velocity of the lymphocyte increases as it approaches the contraction region, the time step of the iteration procedure $(\Delta t)$ is changed depending on the lymphocyte location and is defined as $\Delta t=0.1 \Delta z / u_{\mathrm{WBC}, \max }$, where $u_{\mathrm{WBC}, \max }$ describes the maximum velocity at each time period.

\section{Experimental methods}

\subsection{Microchannel}

The microchannel (Fig. 3) was manufactured using PDMS (poly-dimethylsiloxane) and was prepared using SU-8 (MicroChem Co.) as a casting mold fabricated by lithography. Once removed from the SU-8 mold, the PDMS channel was attached to a cover glass. The working fluids were applied to the inlet of the microchannel by a pump that was driven by pressurized air, and the flow rate was monitored using a micro-flow meter (Sensirion AG.: LG16-0025). The contraction region was located at the midpoint of the streamwise direction. Three inlets were applied to the upstream side to generate a sheath flow, which was used to control the position of the lymphocyte so that it remained located along the channel centerline.

The motion and shape of the lymphocytes were measured using a high-speed video camera (Vision Research Co. Inc., Phantom V7.3). A 100 magnification objective lens (Olympus Co., LMPLFLN100X) was used at a depth of field of $1.7 \mu \mathrm{m}$. The image recoded by the camera had a size of $800 \times 600$ pixels $^{2}$ with a resolution of $0.217 \mu \mathrm{m} /$ pixel. 


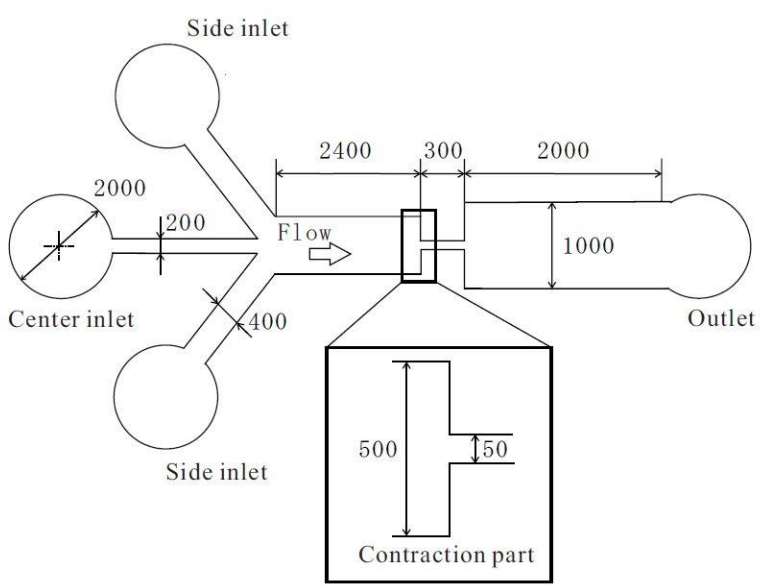

Fig. 3: Structure and dimensions of the microchannel.

\subsection{Sample preparation}

Jurkat cells (ATCC, CRL-2570) were cultured and used as lymphocytes in the experiment. The cultured cells suspended in the $100 \mu \mathrm{L}$ culture medium were centrifuged for $2 \mathrm{~min}$ at $1000 \times g$, and the supernatant was removed. The cell pellet was washed once using PBS (Amresco, E404) and subsequently resuspended in PBS supplemented with PVP (MW $=3.6 \times 10^{5}$, Nakalai tesque, K-90). Solutions of PVP ( $8 \mathrm{wt} \%$ and $12 \mathrm{wt} \%$ ) were used to carry out trial measurements of solutions with different viscosities. By using these two solutions, a wide range of stress conditions generated in the flow were able to be examined in the experiment accurately.

\subsection{Flow conditions}

The rheological properties of these fluids were measured by a rheometer (Anton Paar, MCR 301). The relationship between the viscosity and shear rate is shown in Eq. (3):

$$
\mu_{m}=\left\{\begin{array}{cc}
-0.0711 \log \dot{\gamma}+0.390 \mathrm{~Pa} \cdot \mathrm{s}, & \left(\dot{\gamma}>150 \mathrm{~s}^{-1}\right) \\
0.23 \mathrm{~Pa} \cdot \mathrm{s}, & \left(\dot{\gamma}<150 \mathrm{~s}^{-1}\right)
\end{array}\right.
$$

Thus, zero-shear viscosity of the fluid $\mu_{\mathrm{m}}$ was $0.23 \mathrm{~Pa} \cdot \mathrm{s}$.

The flow rate was varied in the experiment between 0.2 to $2.4 \mu \mathrm{L} / \mathrm{min}$. As mentioned in Section 3.2 , PVP $8 \mathrm{wt} \%$ and $12 \mathrm{wt} \%$ solutions were used to measure the deformation degree, and the flow rate of these two cases was set so that the measurement could be carried out in a wide range of stresses. The flow rates of the PVP $8 \mathrm{wt} \%$ and $12 \mathrm{wt} \%$ solutions were $0.2-2.4$ and $0.2-1.88 \mu \mathrm{L} / \mathrm{min}$, respectively.

\section{Results and discussion}




\subsection{Lymphocyte deformation in the contraction region}

The lymphocyte deformation as it approached and passed the contraction region in the case of ND model is presented in the $x-y$ and $x-z$ planes in Fig. 4. As the lymphocyte approaches the contraction region $(x=0)$ when observed in the $x-y$ plane, it stretched in the streamwise direction and contracted in the spanwise direction. The $x-z$ plane presents a slightly different view, and emphasized the shear stress effect caused by the top and bottom walls of the channel, which demonstrates that shear flow had a greater effect on deformation than the elongational flow.
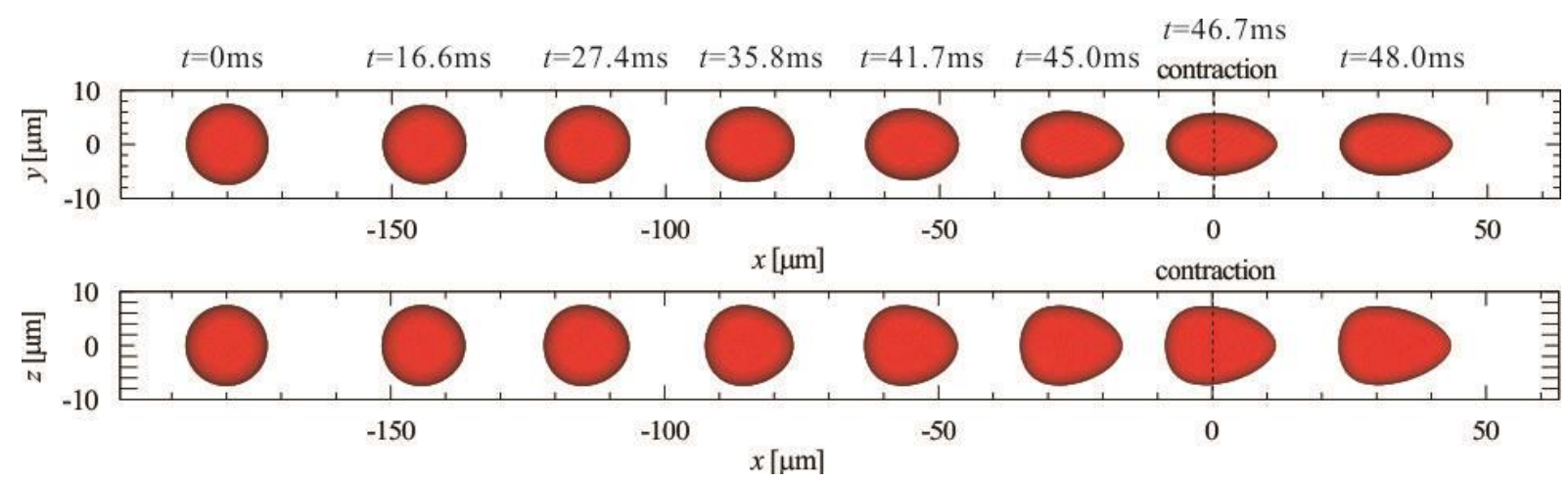

Fig. 4: Projection of the lymphocyte in the $x-y$ and $x-z$ planes showing the deformation of the lymphocyte as it flows through the contraction region obtained by the computation.

\subsection{Effect of flow rate on deformation}

The non-linear characteristics of the lymphocyte deformability were analyzed. The influence of the deformation speed on deformation characteristics was considered by changing the flow rate conditions. Figure 5 shows the relationship between the flow rate $(Q)$ and the deformation index (DI). $D I$ is defined as $D I=\left(l_{x}-l_{y}\right) /\left(l_{x}+l_{y}\right)$, where $l_{x}$ and $l_{y}$ are the lengths of the lymphocyte in the $x$ and $y$ dimensions, respectively. The $D I$ shown in Fig. 5 represents the value of the lymphocyte located at the streamwise position of $x=0$.

In the measurements, as $Q$ increased, $D I$ increased due to the increase of elongational flow and shear stress. The increased rate, however, decreased with $Q$, and the $D I$ shows an asymptotic behavior. The lines show the numerical results of ND, ND+PVP, and STD+PVP models. The ND+PVP and STD+PVP both take into account the shear-thinning effects of the PVP solution in the computation. In the case of ND model, $D I$ remained constant as $Q$ increased. This is attributed to the fact that the streamwise velocity $u_{\mathrm{WBC}}$ and the stress $\sigma_{\text {ext }}$ both increased proportionally with the flow rate. Since the characteristics of cell deformation of the ND model were predominantly viscous, the shape of the cell is defined by the integrated value of the product of $u_{W B C}$ and $\sigma_{\text {ext }}$, and is constant with $Q$. In the ND+PVP model, the $D I$ decreased moderately as $Q$ increased. The 
viscosity of the surrounding fluid decreased as the shear rate of the flow increased due to the shear-thinning effect of the PVP solution and, therefore, the stress applied to the lymphocyte deceased as $Q$ increased.

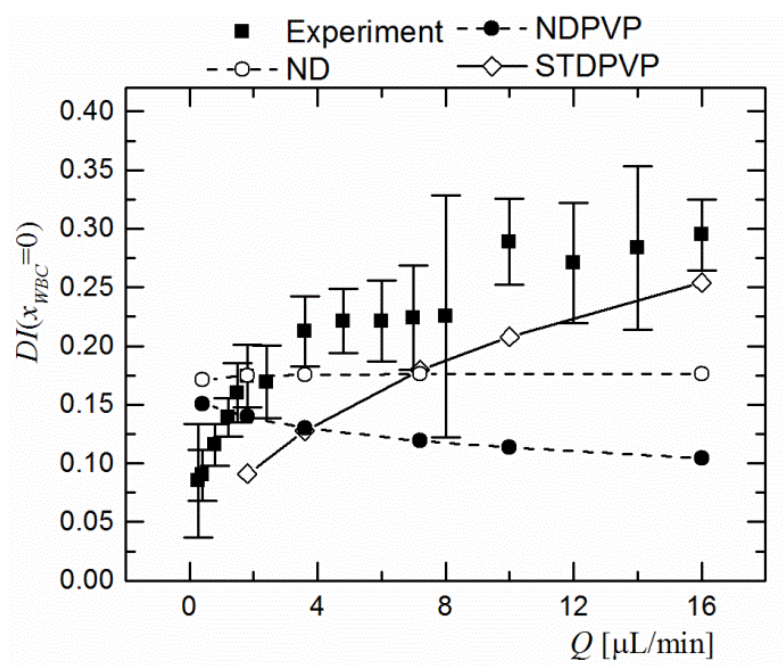

Fig. 5: Relationship between the deformation index $(D I)$ and flow rate $(Q)$. ND model (open circles), cytoplasm is treated as Newtonian fluid; NDPVP model (closed circles), ND model incorporating shear-thinning effects of the surrounding fluids; STDPVP model (open diamonds), shear-thinning effects of the cytoplasm and surrounding fluids are considered. The closed square symbols show the experimental results, the error bars of which are calculated from an uncertainty analysis based on the ASME standard [29].

In both ND and ND+PVP simulations, the distributions were poorly associated with the experimental dataset. On the other hand, the STD+PVP model, which applies a shear-thinning effect to the cytoplasm, showed good agreement with the experimental data in that the DI increased as $Q$ increased, and the rate decreased as $Q$ increased.

The underestimated $D I$ of the STD+PVP model, when compared with the experimental data, is believed to be related to the initial shape of the cell. Figure 6 shows the relationship between the $D I$ and streamwise position of the lymphocyte $\left(x_{\mathrm{WBC}}\right)$ at a $Q$ of $10 \mu \mathrm{mL} / \mathrm{min}$. At each $x_{\mathrm{WBC}}$, the $D I$ of the experimental data showed larger values than that the simulations, particularly in the case of $D I_{0}=0$, despite the observation that the simulated and experimentally derived distributions followed the same trend. Since the lymphocyte showed viscoelastic characteristics when deforming, the shape at any defined moment relies not only on the instantaneous force working on the cell, but also on its shape history. The initial shape can, therefore, have a large effect on the $D I$ distributions thereafter.

To address this, the initial shape of the lymphocyte was changed from spherical to ellipsoid to 
simulate an event by which deformation had already occurred. To define the ellipsoidal shape, we assumed that the cell volume was constant and the lymphocyte would not deform in the height direction. The shape was adjusted by matching the $D I$ at $x_{\mathrm{WBC}}=-83 \mu \mathrm{m}$ of the simulation with the experimental one. The line of $D I_{0}=\Delta D I$ (Fig. 6; chain line) follows the same line of $D I_{0}=0$, except that it has shifted along the vertical axis. The distribution of the data is consistent with the experimental data, particularly in the region located upstream of the contraction. These distributions not only explain the deviation, but also show the validity of the present model for predicting the deformation of lymphocytes.

Some deviation still remains at the contraction and downstream regions $(x>0)$, which is believed to be attributed to the elastic properties of the lymphocyte and the influence of the nucleus. The membrane structure shows elasticity and the nucleus will suppress the deformation (as described below). In order to evaluate these effects, it would be necessary to measure the steady state deformation under a constant shear rate.

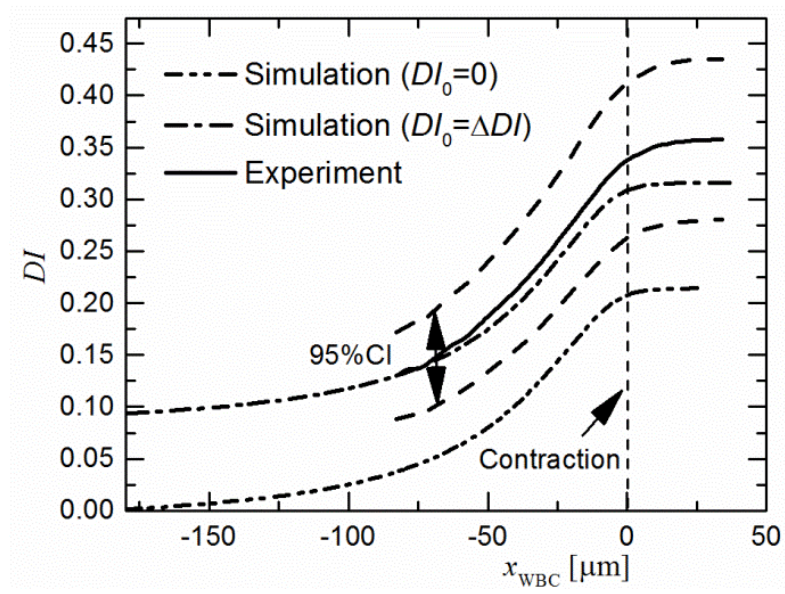

Fig. 6: Relationship between the deformation index $(D I)$ and streamwise location of the lymphocytes, where $Q=10 \mu \mathrm{L} / \mathrm{min}$. The solid and dotted lines with black color depict the average value and the $95 \%$ uncertainty range of the experimental results, respectively.

\subsection{Effect of the nucleus}

The uncertainty of the experiment, which is depicted by the large error bars in Fig. 5, is large and is believed to be attributed to a number of variables, including the dispersion of the cell size, cell position in the flow, the properties of the cells, and nucleus size and position. To address some of these variables, the position of the nucleus in the cell and its influence on cell deformation, and the flow pattern generated in the cytoplasm is analyzed. The velocity vector distributions in the lymphocyte in the cross-sectional plane of $z=0$ is presented in Fig. 7. The flow rate was defined as $Q=1.8 \mu \mathrm{l} / \mathrm{min}$ and the results show the moment when the lymphocytes were positioned at 
$x_{\mathrm{WBC}}=0 \mu \mathrm{m}$. The vectors are for velocities relative to the center of mass of the cell. Figure (a) shows the STD case, and (b) shows the STCD case.

In the STD model (Fig. 7 (a)), the flow in the cell tends to be very similar to the flows observed in simple elongational flow. On the other hand, in the STCD model (Fig. (b)), the flow pattern is significantly different to the STD model; since the viscosity of the nucleus is high, it did not deform as the cell flowed through the contraction region under the conditions considered in this study. Due to the rigidity of the nucleus, the flow in the cell was interrupted; the relative velocity near the nucleus was zero and increased with distance to the nucleus. Therefore, the cell membrane easily deformed when it was located away from the nucleus, whereas it became less deformable in the area adjacent to the nucleus. This led to the suppression of deformation in the spanwise direction and trailing side of the lymphocyte in the area in which the membrane was in close proximity to the nucleus. An asymmetric deformation in the streamwise direction is thus observed in the STCD case.

The influence of the nuclear streamwise position on the lymphocyte deformation is shown in Fig. 8. In Fig. 8 (b), the leading side of the lymphocyte was deformed and stretched in the streamwise direction, while the deformation at the trailing side was small and negligible. In Fig. 8 (a), when the nucleus was located closer to the trailing side, the leading side was largely deformed when compared with (b). When the nucleus was located closer to the leading side as shown in Fig. 8 (c), the deformation was largely suppressed at the leading side while the increase of deformation at the trailing side was moderate. These effects are evident in the experimental flow, of which snapshots of two samples are presented in Fig. 9. The tendency of the nucleus to affect the deformation properties was consistent with the simulated data in Fig. 8. The DI variation in relation to the nucleus position was more than $50 \%$ in the experimental data. This is comparative with the magnitude of the error bars shown in Fig. 5 and the confidence intervals shown in Fig. 6 , indicating that the position of the nucleus is a significant factor responsible for the variation in deformation characteristics of a lymphocyte in a flow.

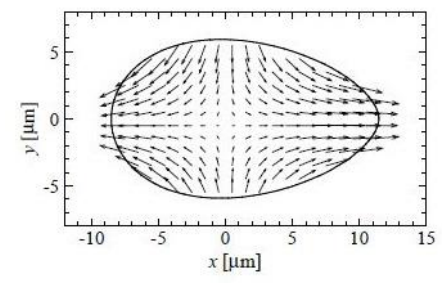

(a) STD model

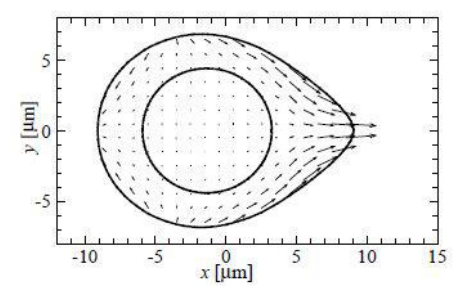

(b) STCD model

Fig. 7: Effect of the nucleus on the lymphocyte deformation at $x_{\mathrm{WBC}}=0 \mu \mathrm{m}$ (velocity vectors show the flow in the lymphocyte). 


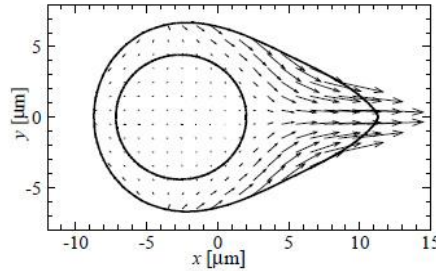

(a) $\Delta x_{\mathrm{n}}=-1.5$

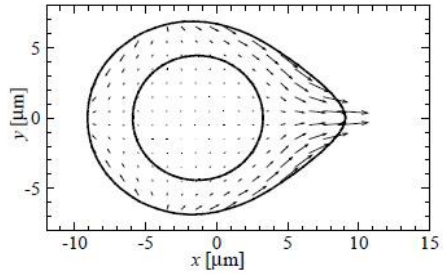

(b) $\Delta x_{\mathrm{n}}=0$

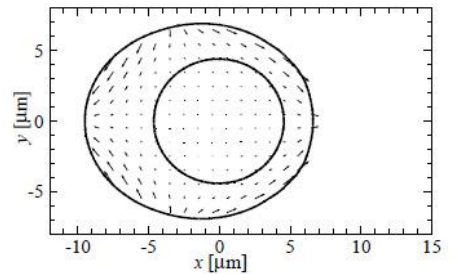

(c) $\Delta x_{\mathrm{n}}=+1.5$

Fig. 8: Effects of the nucleus streamwise position on the lymphocyte deformation. (a) $\Delta x_{n}=-1.5$ $\mu \mathrm{m}$. (b) $\Delta x_{\mathrm{n}}=0 \mu \mathrm{m}$. (c) $\Delta x_{\mathrm{n}}=1.5 \mu \mathrm{m}$.

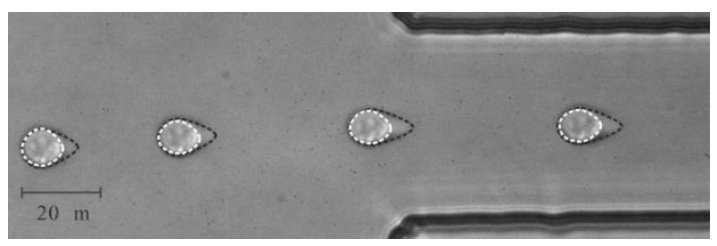

(a) Nucleus located at trailing side

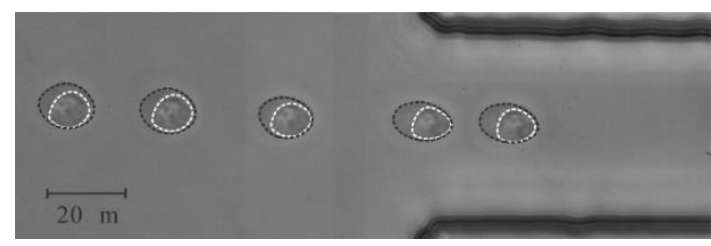

(b) Nucleus located at leading side

Fig. 9: Influence of the nucleus position on lymphocyte deformation. (a) Nucleus located at the trailing side of the lymphocyte. (b) Nucleus located at the leading side of the lymphocyte. The location of the nucleus is estimated and is depicted by the white dash lines in the photographs.

\subsection{Properties of lymphocyte viscosity}

The results presented demonstrate how the shear-thinning effects and the nucleus need to be considered in order to obtain an accurate prediction of the deformation characteristics of the lymphocytes. Given that there was good agreement between the computational prediction and experimental validation shown in Fig. 5, it was therefore possible to adjust the model to measure the viscosity of the lymphocyte.

A schematic of the computation of lymphocyte deformation in a simple elongational flow is shown in Fig. 10. Using the ND model in such a simple condition, the deformation rate is proportional to the shear rate and the length of the cell. Therefore, the length of the lymphocyte in the $x$ direction $\left(l_{x}\right)$ and the shear rate of the fluid $(\dot{\gamma})$ will show the following relationship:

$$
i_{x}=2 \dot{l}_{x}
$$

Using the cell diameter $\left(a_{c}\right)$ as the initial length and time $(t), l_{x}$ can be described as:

$$
l_{x}=a_{c} e^{\dot{\gamma}}
$$

Therefore, $D I$ can be described as: 


$$
\begin{aligned}
D I & =\frac{a_{c} e^{\dot{\gamma} t}-a_{c} e^{-\dot{\gamma} t}}{a_{c} e^{\dot{\gamma} t}+a_{c} e^{-\dot{\gamma} t}} \\
& =\tanh \dot{\gamma} t \\
& \approx \dot{\gamma} t
\end{aligned}
$$

The relationship between the elapsed time $(t)$ and the $D I \cdot \lambda$ is presented in Fig. 11 , where $\lambda$ is ratio of the fluid viscosity $\mu_{\mathrm{m}}$ and lymphocyte viscosity $\mu_{\mathrm{c}}: \lambda=\mu_{\mathrm{c}} / \mu_{\mathrm{m}}$. A linear relation is observed between $t$ and $D I \cdot \lambda$ for each case of $\lambda$. Further, as $\lambda$ increases, the lines converge to one line. When $\lambda=1$, the fluid in the lymphocyte will trace the elongational flow, that is, the overall flow pattern will form as if no object is inserted. As $\lambda$ increases, the flow will be affected by the existence of the lymphocyte. When $\mu_{\mathrm{c}}$ becomes sufficiently large, then the problem can be simplified as the balance between the stress applied to the lymphocyte by the surrounding fluid and the stress required for the deformation, i.e. $\dot{D} I \cdot \lambda=\dot{\gamma}$.

Considering then that the ratio of the slope of each line in the cases of $\lambda=1$ and 50 is 2.96 , the following relationship can be assumed:

$$
\begin{aligned}
\dot{D} I & =2.96 \frac{\dot{\gamma}}{\lambda} \\
& =2.96 \frac{\mu_{m} \dot{\gamma}}{\mu_{c}} \\
& =0.74 \frac{\sigma_{e x t}}{\mu_{c}}
\end{aligned}
$$

Where $\sigma_{\text {ext }}$ is the apparent stress generated by the elongational flow. Since the $\lambda$ in the experiment is greater than 50, Eq. (7) can be applied at each streamwise position. Therefore, Eq. (7) can be described as:

$$
\dot{D} I(x)=0.74 \frac{\sigma_{e x t}(x)}{\mu_{c}}
$$

These relationships are for Newtonian fluids, and in the case of the PVP solution, which causes the shear-thinning effects to appear, Eq. (8) should be modified by applying the function $f(\gamma)$ :

$$
\dot{D} I(x)=0.74 \frac{\sigma_{e x t}(x)}{\mu_{c}} f(\dot{\gamma})
$$

In order to obtain $f(\gamma)$, the elongational flow using the PVP solution is computationally determined. From these results, the relationship between $D I(x) \mu_{\mathrm{c}} / 0.74 \sigma_{\mathrm{ext}}(x)$ and $\gamma$ is derived as shown in Fig. 12. Therefore, $f(\gamma)$ can be defined as: 


$$
f(\dot{\gamma})=\left\{\begin{array}{lc}
-0.29576 \log \dot{\gamma}+1.5213, & \left(\dot{\gamma}>57.9 \mathrm{~s}^{-1}\right) \\
1, & \left(\dot{\gamma}<57.9 \mathrm{~s}^{-1}\right)
\end{array}\right.
$$

When Eqs. (9) and (10) are applied to the experiment, the viscosity $\mu_{\mathrm{c}}$ can be calculated using $\sigma_{\mathrm{ext}}$, $f(x)$, and $\dot{D} I$. $\dot{D} I$ is measured in the area of $-50 \mu \mathrm{m}<x \mathrm{wBC}<0 \mu \mathrm{m}$, and the value $\sigma_{\text {ext }}(x)$ is derived from the numerical results of the flow. Figure 13 shows the relationship between $\mu_{\mathrm{c}}$ and $\dot{\gamma}$. For comparison, the results of Eq. (1), to which the values of Drury et al. [24] are applied, are shown by the dashed line. The results of the computation using the STD model, where the shearthinning effects, are shown by the filled circles.

The measurement and numerical results matches well with each other, and linearly decreases under all flow rate conditions with the same tendency of Drury et al. This confirms that a simplified model can be applied to measure the shear-thinning effect of the viscosity of the lymphocyte.

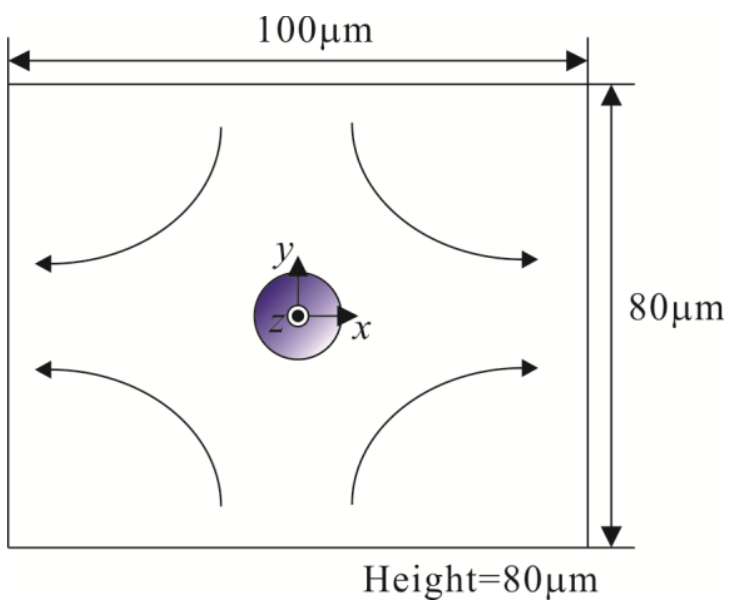

Fig. 10: Computational domain of the lymphocyte in steady elongational flow. 


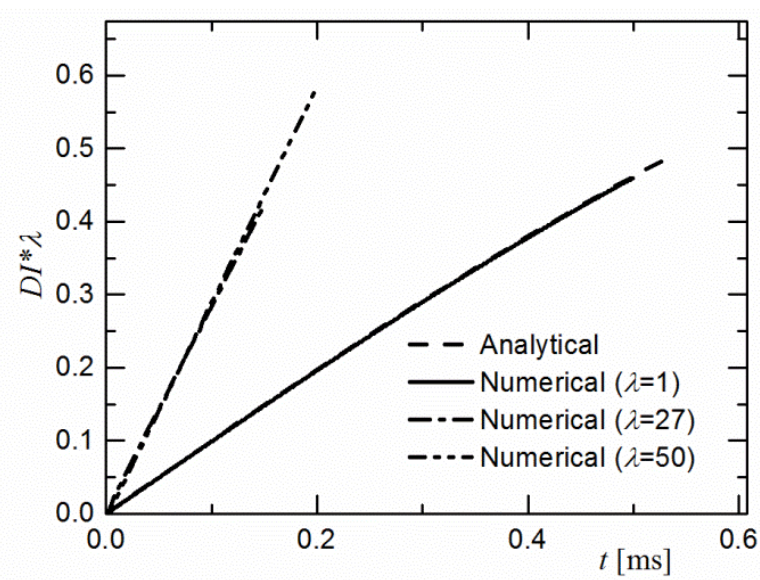

Fig. 11: Time-dependent distributions of deformation index $(D I)$ of the lymphocyte in steady elongational flow under different viscosity ratios

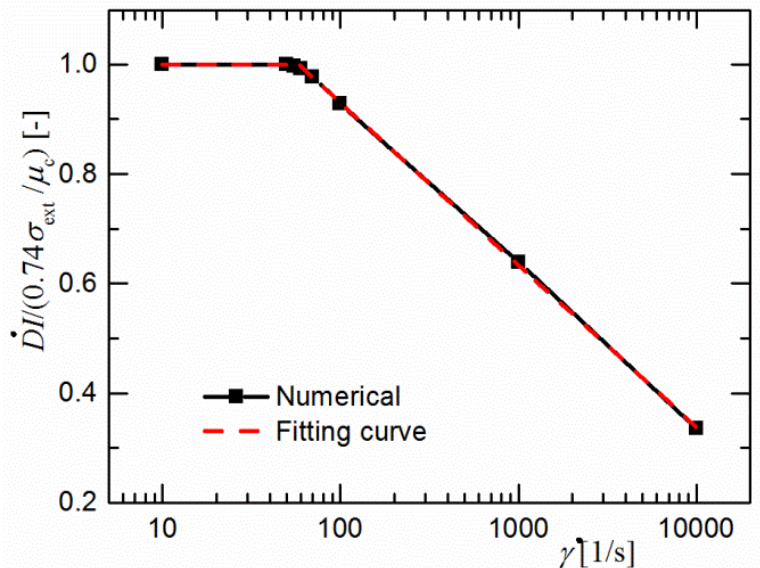

Fig. 12: Relationship between time differential value of deformation index $(D I)$ and shear rate $\dot{\gamma}$ in the case of steady elongational flow considering the shear-thinning effect of the PVP solution.

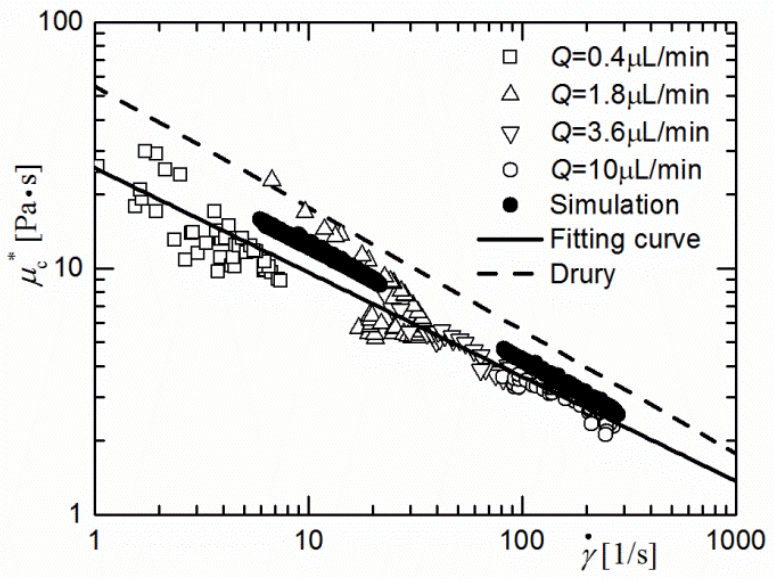


Fig. 13: Relationship between the apparent lymphocyte viscosity $\mu_{\mathrm{c}}$ and shear rate $\dot{\gamma}$.

On the other hand, the experimental and simulated results were smaller than the results of Drury et al [24]. Three reasons for this differentiation are proposed: firstly, there is an assumption that the shear rate is uniform throughout the lymphocyte. Secondly, once the lymphocyte is deformed for a certain time, the influence of the nucleus cannot be negligible. Finally, due to the top and bottom walls, the shear flow effect appears in the $z$ direction. The shear stress is smaller compared to the elongational stress as shown by the smaller deformation in the $z$ direction in Fig. 4, and therefore, $D I$ based on the lymphocyte shape in the $x-y$ plane may be overestimated.

Reasonable results have, thus, been obtained. However, nucleus size, shape and position influences the flow and suppresses the deformation of the lymphocyte as discussed in Section 4.3. Therefore, a certain amount of uncertainty will always exist in the measurement of the cell deformation. The deformability can change depending on the deformation rate since the influence of the nucleus on the flow of the cytoplasm is dependent on the distance between the cell membrane and the nucleus. If these effects are to be applied to a simplified equivalent model, the model needs to be improved, for example, by applying multi-stage characteristics of the viscoelastic properties to the equivalent model.

\section{Conclusions}

In this study, a numerical approach based on a drop model to characterize properties of lymphocytes in flow conditions is described. The deformation characteristics of a cell passing through a contraction region in a microchannel were calculated and experimentally validated. The new model provided significant improvements over the conventional drop model, as shown by the experimentally derived data, through the application of shear-thinning effects to the cytoplasm, and by incorporating properties of the nucleus. The presence of the nucleus was found to suppress the deformation of the cell, particularly in the span-wise direction. A slight deviation in the position of the nucleus changed the shape of the cell markedly, resulting in uncertainty in the DI. Finally, the apparent viscosity of the lymphocyte was derived by applying the simplified model, which was based on a lymphocyte deformation in a steady elongational flow, to the measurement. The results showed a good performance in predicting the shear-thinning effects covering a wide range of shear rates. 


\section{References}

[1] Gregg, E. C. and Steidley, K. D.: Electrical Counting and Sizing of Mammalian Cells in Suspension. Biophysical Journal. 5, 393-405 (1965)

[2] Morgan, H., Sun, T., Holmes, D., Gawad, S. and Green, N. G.: Single Cell Dielectric Spectroscopy. J. Physics D: Applied Physics. 40, 61-70 (2007)

[3] Schade-Kampmamm, G., Huwiler, A., Hebeisen, M., Hessler, T., and Berardino, M. D.: On-chip Non-invasive and Label-free Cell Discrimination by Impedance Spectroscopy. Cell Proliferation. 41, 830-840 (2008)

[4] Perktold, K., and Rappitsch, G.: Computer Simulation of Local Blood Flow and Vessel Mechanics in a Compliant Carotid Artery Bifurcation Model. Journal of Biomechanics. 28, 845-856 (1995)

[5] Taylor, C. A., Hughes, T. J. R., and Zarins, C. K.: Finite Element Modeling of Blood Flow in Arteries. Computer Methods in Applied Mechanics and Engineering. 158, 155-196 (1998)

[6] Baumler, H., Neu, B., Donath, E., and Kiesewetter, H.: Basicphenomena of Red Blood Cell Rouleaux Formation. Biorheology. 36, 439-442 (1999)

[7] Chen, J., and Lu, X.-Y.: Numerical Investigation of the Non-Newtonian Pulsatile Blood Flow in a Bifurcation Model with a Non-Planar Branch. Journal of Biomechanics. 39, 818832 (2006)

[8] Bagchi, P.: Mesoscale Simulation of Blood Flow in Small Vessels. Biophysical Journal. 92, 1858-1877 (2007)

[9] Wang, T., Pan, T.-W., Xing, Z. W., and Glowinski, R.: Numerical Simulation of Rheology of Red Blood Cell Rouleaux in Microchannels. Physical Review E. 79, 041916 (2009)

[10] Grinberg, L., Cheever, E., Anor, T., Madsen, J. R., and Karniadakis, G. E.: Modeling Blood Flow Circulation in Intracranial Arterial Networks: A Comparative 3D/1D Simulation Study. Annals of Biomedical Engineering. 29, 297-309 (2011)

[11] Mahalingam, M., Pozniak, A., Mcmanus, T. J., Vergani, D. and Peakman, M.: Cell Cycling HIV Infection: Analysis of In Vivo Activated Lymphocytes. Experimental Immunology. 102, 481-486 (1995)

[12] Wurtzer, S., Compain, S., Benech, H., Hance, A. J. and Clavel, F.: Effect of Cell Cycle Arrest on the Activity of Nucleoside Analogues against Human Immunodeficiency Virus Type 1. J. Virology. 79, 14815-14821 (2005)

[13] Roda, B., Reschinglian, P., Zattoni, A., Luigi, P., Buzzi, M., Ricci, F. and Bontadini, A.: Human Lymphocyte Sorting by Gravitational Field-flow Fractionation. Analytical and Bioanalytical Chemistry, 392, 137-145 (2008)

[14] Schmid-Schönbein, G. W., Sung, K. P., Tözeren, H., Skalak, R., and Chien, S.: Passive 
Mechanical Properties of Human Leukocytes, Biophysical J. 36, 243-256 (1981)

[15] Tsai, M. A., Frank, R. S., and Waugh, R. E.: Passive Mechanical Behavior of Human Neutrophils: Power-law Fluid. Biophysical Journal. 65 2078-2088 (1993)

[16] Kan, H. C., Udaykumar, H. S., Shyy, W. and Tran-Son-Tay, R.: Hydrodynamics of a Compound Drop with Application to Leukocyte Modeling. Physics of Fluids. 10, 760-774 (1998)

[17] Evans, E. and Needham, D.: Physical Properties of Surfactant Bilayer Membranes: Thermal Transitions, Elasticity, Rigidity, Cohesion, and Colloidal Interactions. J. Physical Chemistry. 91 4219-4228 (1987)

[18] Vigneron, P., A thesis presented to the graduate school of the University of Florida, University of Florida. (1998)

[19] Dong, C. and Skalak, R.: Leukocyte Deformability: Finite Element Modeling of Large Viscoelastic Deformation. Journal of Theoretical Biology. 158, 173-193 (1992)

[20] Schmid-Schönbein, G. W., Shih, Y. Y. and Chien, S.: Morphometry of Human Leukocytes. Blood. 56, 866-875 (1980)

[21] Luo, Z. Y., Xu, F., Lu, T. J., and Bai, B. F.: Direct Numerical Simulation of Single Leukocyte Deformation in Microchannel Flow for Disease Diagnosis. J. Medical Systems. 35, 869-876 (2011).

[22] Kan, H-C, Shyy, W., Udaykumar, H. S., Vigneron, P., and Tran-Son-Tay, R.: Effects of Nucleus on Leukocyte Recovery. Annals of Biomedical Engineering. 27, 648-655 (1999)

[23] Khismatullin, D. B., and Truskey, G. A.: Three-dimensional Numerical Simulation of Receptor-Mediated Adhesion to Surfaces: Effects of Cell Deformability and Viscoelasticity. Physics of Fluids. 17, 031505 (2005)

[24] Drury, J. L. and Dembo, M.: Aspiration of Human Neutrophils: Effects of Shear Thinning and Cortical Dissipation. Biophysical Journal. 81, 3166-3177 (2001)

[25] Peskin, C. S.: Numerical Analysis of Blood Flow in the Heart. J. Computational Physics 25, 220-252 (1977)

[26] Yeung, A. and Evans, E.: Cortical Shell-liquid Core Model for Passive Flow of Liquid-like Spherical Cells into Micropipettes. Biophysical Journal. 56, 139-149 (1989)

[27] Needham, D. and Hochmuth, R. M.: A Sensitive Measure of Surface Stress in the Resting Neutrophil. Biophysical Journal. 61, 1664-1670 (1992)

[28] Kaleridis, V., Athanassiou, G., Deligianni, D. and Missirlis, Y.: Slow flow of Passive Neutrophils and Sequestered Nucleus into Micropipette. Clinical Hemorheology and Microcirculation. 45, 53-65 (2010)

[29] ASME. Measurement Uncertainty, Performance Test Codes ANSI/ASME PTC 19.1-1985. ASME, New York (1986) 


\section{Acknowledgments}

This work was supported by Japan Society for the Promotion of Science (JSPS) KAKENHI Grant Number 24360080. 